\title{
The Differential Impact of COVID-19 on Urban Versus Rural Dermatologic Practice Logistics and Recovery: A Cross-Sectional Investigation of the First Wave
}

\author{
Justin W. Marson, MD¹, Graham H. Litchman, DO, $\mathrm{MS}^{2}$, Darrell S. Rigel, MD, MS ${ }^{3}$ \\ ${ }^{1}$ National Society for Cutaneous Medicine, New York, NY \\ 2Department of Dermatology, St. John's Episcopal Hospital, New York, NY \\ ${ }^{3}$ Department of Dermatology, NYU Grossman School of Medicine, New York, NY
}

\section{ABSTRACT}

Background: COVID-19 materially delayed patient visits and potential skin cancer biopsies/diagnoses among US dermatology practices. However, given a likely heterogenous impact across the US, this study sought to determine COVID-19's effect on urban versus rural dermatology practices.

Methods: Data were analyzed from the first 1000 responses to 3 pre-validated surveys of 9891 practicing US dermatologists comparing outpatient volumes and scheduling issues for the week of February 17th to the week of March $16^{\text {th }}$ (Survey 1), April $13^{\text {th }}$ (Survey 2) and May $18^{\text {th }}, 2020$ (Survey 3). First 3 US zip-code digits were compared to US Census Bureau data to determine "Urban/Rural" status. Representativeness with AAD membership was confirmed. Statistical significance was calculated using chi-square with Marascuilo procedure and two-tailed independent t-test/ANOVA with post-hoc Tukey-Kramer testing.

Results: In April 2020 urban practices reported more closed practices (21.4\% vs 5.8\%, p<0.0001) and predicted significantly larger patient volume decreases $(-45.2 \%$ vs $-31.4 \%, p<0.0001)$ and practice closures (11.9\% vs. $2.5 \%$ p $<0.0001)$ in the following 2 weeks. In May 2020, urban areas saw significantly fewer patients/week (90.9 vs $142.4 p<0.0001)$, larger decrease in patient volume relative to May $2019(-49.4 \%$ vs $-35.1 \%, p<0.0001)$, and conducted more telemedicine visits $(27.0 \%$ vs $15.1 \%, p<0.0001)$. Significantly more rural practices reported already being at baseline volume (Mean Difference $6.2 \%, 95 \% \mathrm{Cl} 2.7 \%-9.8 \%$ ) while urban practices predicted return to baseline volume by August $(5.7,95 \% \mathrm{Cl} 2.1 \%-9.3 \%)$ or were unsure $(5.6,95 \% \mathrm{Cl} 1.6 \%-9.7 \%)$.

Conclusion: The initial COVID-19 pandemic differentially affected urban dermatology practices. The effects of the pandemic were mitigated in part by increased use telemedicine. Future studies may further elucidate COVID-19's effect on clinical practice and highlight areas for improvement in practice logistics and patient care.

\section{INTRODUCTION}

The COVID-19 pandemic has significantly affected clinical practice worldwide. ${ }^{1,2}$ However, the degree to which US regions and therefore local healthcare systems were affected varied during the initial pandemic. ${ }^{3}$ The purpose of this study was to determine the COVID-19 pandemic's differential impact on dermatologic outpatient care in urban versus rural areas in the United States.

March 2021 Volume 5 Issue 2 


\section{METHODS}

Data were analyzed from the first 1000 responses to 3 pre-validated surveys of 9891 practicing US dermatologists collected for the week of February $17^{\text {th }}$, March $16^{\text {th }}$ (Survey 1), April $13^{\text {th }}$ (Survey 2) and May $18^{\text {th }}$, 2020 (Survey 3). Results compared outpatient volumes and scheduling issues between these time points. The first 3 digits of US zip codes (section code) were compared to US Census Bureau data ${ }^{4}$ to determine "Urban/Rural" status. Section codes containing counties with both designations were assigned based on predominate designation within that region. Representativeness with AAD membership was confirmed with 95\% confidence intervals (Cl) (Tables 1,2). Statistical significance was calculated using chi-square with Marascuilo procedure for categorical data and two-tailed independent t-test with unequal variance/ANOVA with post-hoc Tukey-Kramer testing for continuous data.

\section{RESULTS}

There were significantly more urban respondents in regions with section codes beginning with 1,8 and 9 and more rural in regions 2,4, and 5 (Table 1). Practice mix/type was similar between urban/rural practices and across surveys (Table 2). In April 2020 urban practices predicted significantly larger patient volume decreases over the next 2 weeks(-45.2\%; $95 \% \mathrm{Cl}-47.9$ to -42.4 vs $-31.4 \% ; 95 \% \mathrm{Cl}-37.9$ to -24.9 , $\mathrm{p}<0.0001$ ), and practice closures in the following 2 weeks $(11.9 \%$; $9.7 \%-14.1 \%$ vs $2.5 \% ; 0.0 \%-5.4 \%, p<0.0001)$ and reported more closed practices $(21.4 \% ; 18.6 \%-24.1 \%$ vs $5.8 \% ; 1.6 \%-10.1 \%, p<0.0001$ ) (Table 3 ). Urban practices also obtained fewer biopsies of suspicious-pigmented lesions (2.7;2.4-3.1 vs 4.8;3.4-6.2) however this finding only trended towards significance after post-hoc testing.

In May 2020, both urban and rural practices predicted similar increases in practice volume. However, urban areas saw significantly fewer patients/week (90.9; 81.6 - 100.2 vs $142.4 ; 105.0-179.8, \mathrm{p}<0.0001)$ and larger decreases in patient volume relative to May $2019(-49.4 \% ;-51.2 \%$ to $47.6 \%$ vs $-35.1 \% ;-39.6 \%$ to $-30.6 \%$, $\mathrm{p}<0.0001)$. Furthermore, a significantly larger proportion of rural practices reported being already back to baseline volume compared to urban practices (Mean Difference $\quad 6.2 \%, \quad 2.7 \%-9.8 \%$ ). Urban practices had a median predicted to baseline patient volumes by August $(5.7 \%$, $2.1 \%-9.3 \%)$ or were unsure $(5.6 \%, 1.6 \%$ $9.7 \%$ ) (Table 4). Urban practices conducted a significantly higher percentage of visits via telemedicine (27.0\%; $24.7 \%-29.3 \%$ vs $15.1 \% ; 10.3 \%-19.8 \%, p<0.0001)$. Urban areas also predicted a higher percentage of telemedicine usage in June 2020 (21.8\%; $19.9 \%-23.7 \%$ vs $12.2 \%$; $9.1 \%-15.4 \%$ ).

\section{DISCUSSION}

The initial COVID-19 pandemic had a heterogeneous impact on dermatologic practices, wherein urban practices experienced significantly more practice losses and a slower recovery. These results suggest "hot-spots" likely played a larger role than dermatology density in modulating relative impact as more heavily populated/urban areas had more active cases and generally stricter governmental/institutional regulations earlier in the pandemic. ${ }^{3,5}$ The recovery pattern 


\section{SKIN}

Table 1. Geographic representation by Urban/Rural designation compared to AAD membership data. Per survey, overall regional demographics congruent with national data. Notably, increased proportions of respondents from urban areas in regions 1,8 and 9 while there were increased proportions of dermatologists in rural areas in regions 2,4 and 5

\begin{tabular}{|c|c|c|c|c|c|c|c|c|c|c|}
\hline & & $\begin{array}{c}\text { Survey } 1 \\
\%(95 \% \mathrm{Cl})\end{array}$ & & & $\begin{array}{c}\text { Survey } 2 \\
\%(95 \% \mathrm{Cl})\end{array}$ & & & $\begin{array}{l}\text { Survey } 3 \\
\%(95 \% \mathrm{Cl})\end{array}$ & & p-value \\
\hline $\begin{array}{l}\% \text { AAD US } \\
\text { Membership }\end{array}$ & Overall & $\begin{array}{c}\text { Urban } \\
(n=847)\end{array}$ & $\begin{array}{c}\text { Rural } \\
(n=118)\end{array}$ & Overall & $\begin{array}{l}\text { Urban } \\
(n=875)\end{array}$ & $\begin{array}{c}\text { Rural } \\
(n=120)\end{array}$ & Overall & $\begin{array}{l}\text { Urban } \\
(n=742)\end{array}$ & $\begin{array}{l}\text { Rural } \\
(n=87)\end{array}$ & \\
\hline 9.6 & $\begin{array}{c}9.4 \\
(7.5-11.3)\end{array}$ & $\begin{array}{c}9.9 \\
(7.9-12.0)\end{array}$ & $\begin{array}{c}5.9 \\
(1.6-10.3)\end{array}$ & $\begin{array}{c}8.4 \\
(6.6-10.2)\end{array}$ & $\begin{array}{c}9.3 \\
(7.3-11.2)\end{array}$ & $\begin{array}{c}2.5^{\star \ddagger} \\
(0.0-5.4)\end{array}$ & $\begin{array}{c}9.8 \\
(7.7-11.9)\end{array}$ & $\begin{array}{c}10.1 \\
(7.9-12.3)\end{array}$ & $\begin{array}{c}8.2 \\
(2.3-14.2)\end{array}$ & 0.1524 \\
\hline 12.8 & $\begin{array}{c}14.0 \\
(11.8-16.2)\end{array}$ & $\begin{array}{c}15.7 \\
(13.2-18.2)\end{array}$ & $\begin{array}{c}2.5^{\star \ddagger} \\
(0.00-5.4)\end{array}$ & $\begin{array}{c}15.8 \\
(13.5-18.1)\end{array}$ & $\begin{array}{c}17.0 \\
(14.5-19.6)\end{array}$ & $\begin{array}{c}7.5 \\
(2.7-12.3)\end{array}$ & $\begin{array}{c}17.5 \\
(14.9-20.1)\end{array}$ & $\begin{array}{c}18.4^{\ddagger} \\
(15.5-21.2)\end{array}$ & $\begin{array}{c}11.8 \\
(4.8-18.8)\end{array}$ & $<0.0001$ \\
\hline 10.4 & $\begin{array}{c}11.0 \\
(9.0-12.9)\end{array}$ & $\begin{array}{c}9.2^{*} \\
(7.2-11.2)\end{array}$ & $\begin{array}{c}24.6^{\ddagger} \\
(16.6-32.5)\end{array}$ & $\begin{array}{c}10.9 \\
(8.9-12.9)\end{array}$ & $\begin{array}{c}8.9^{*} \\
(7.0-10.8)\end{array}$ & $\begin{array}{c}25.8^{\ddagger} \\
(17.8-33.8)\end{array}$ & $\begin{array}{c}8.9 \\
(6.9-10.9)\end{array}$ & $\begin{array}{c}7.3 \\
(5.4-9.2)\end{array}$ & $\begin{array}{c}23.5 \\
(14.3-32.7)\end{array}$ & $<0.0001$ \\
\hline 13.8 & $\begin{array}{c}12.3 \\
(10.2-14.4)\end{array}$ & $\begin{array}{c}12.4 \\
(10.1-14.7)\end{array}$ & $\begin{array}{c}11.9 \\
(5.9-17.8)\end{array}$ & $\begin{array}{c}12.4 \\
(10.3-14.5)\end{array}$ & $\begin{array}{c}12.0 \\
(9.8-14.2)\end{array}$ & $\begin{array}{c}15.8 \\
(9.1-22.5)\end{array}$ & $\begin{array}{c}11.5 \\
(9.3-13.7)\end{array}$ & $\begin{array}{c}11.3 \\
(9.0-13.7)\end{array}$ & $\begin{array}{c}14.1 \\
(6.6-21.7)\end{array}$ & 0.3030 \\
\hline 8.4 & $\begin{array}{c}7.6 \\
(5.9-9.3)\end{array}$ & $\begin{array}{c}7.0 \\
(5.2-8.7)\end{array}$ & $\begin{array}{c}12.7 \\
(6.6-18.8)\end{array}$ & $\begin{array}{c}8.6 \\
(6.8-10.4)\end{array}$ & $\begin{array}{c}7.9 \\
(6.0-9.7)\end{array}$ & $\begin{array}{c}14.2 \\
(7.8-20.5)\end{array}$ & $\begin{array}{c}6.3 \\
(4.6-8.0)\end{array}$ & $\begin{array}{c}5.8 \\
(4.1-7.5)\end{array}$ & $\begin{array}{c}11.8 \\
(4.8-18.8)\end{array}$ & 0.0056 \\
\hline 5.0 & $\begin{array}{c}3.9 \\
(2.7-5.1)\end{array}$ & $\begin{array}{c}3.1 \\
(1.9-4.3)\end{array}$ & $\begin{array}{c}10.2 \\
(4.6-15.7)\end{array}$ & $\begin{array}{c}4.2 \\
(2.9-5.5)\end{array}$ & $\begin{array}{c}3.4 \\
(2.2-4.7)\end{array}$ & $\begin{array}{c}10.0 \\
(4.5-15.4)\end{array}$ & $\begin{array}{c}3.7 \\
(2.4-5.0)\end{array}$ & $\begin{array}{c}3.2 \\
(1.9-4.5)\end{array}$ & $\begin{array}{c}8.2 \\
(2.3-14.2)\end{array}$ & $<0.0001$ \\
\hline & $\begin{array}{c}6.6 \\
(5.0-8.2)\end{array}$ & $\begin{array}{c}6.5 \\
(4.8-8.2)\end{array}$ & $\begin{array}{c}7.6 \\
(2.7-12.5)\end{array}$ & $\begin{array}{c}6.2 \\
(4.7-7.7)\end{array}$ & $\begin{array}{c}6.3 \\
(4.6-7.9)\end{array}$ & $\begin{array}{c}5.8 \\
(1.6-10.1)\end{array}$ & $\begin{array}{c}6.6 \\
(4.9-8.3)\end{array}$ & $\begin{array}{c}5.9 \\
(4.2-7.7)\end{array}$ & $\begin{array}{c}12.9 \\
(5.7-20.2)\end{array}$ & 0.3594 \\
\hline 10.0 & $\begin{array}{c}10.0 \\
(8.1-11.9)\end{array}$ & $\begin{array}{c}9.3 \\
(7.3-11.3)\end{array}$ & $\begin{array}{c}14.4 \\
(7.9-20.9)\end{array}$ & $\begin{array}{c}8.7 \\
(6.9-10.5)\end{array}$ & $\begin{array}{c}8.7 \\
(6.8-10.6)\end{array}$ & $\begin{array}{c}9.2 \\
(3.9-14.4)\end{array}$ & $\begin{array}{c}9.9 \\
(7.8-12.0)\end{array}$ & $\begin{array}{c}10.8 \\
(8.5-13.1)\end{array}$ & $\begin{array}{c}3.5 \\
(0.0-7.5)\end{array}$ & 0.1658 \\
\hline 6.2 & $\begin{array}{c}8.4 \\
(6.6-10.2)\end{array}$ & $\begin{array}{c}8.9 \\
(6.9-10.8)\end{array}$ & $\begin{array}{c}5.1 \\
(1.0-9.1)\end{array}$ & $\begin{array}{c}7.7 \\
(6.0-9.4)\end{array}$ & $\begin{array}{c}8.0 \\
(6.2-9.8)\end{array}$ & $\begin{array}{c}5.8 \\
(1.6-10.1)\end{array}$ & $\begin{array}{c}6.6 \\
(4.9-8.3)\end{array}$ & $\begin{array}{c}6.9 \\
(5.0-8.7)\end{array}$ & $\begin{array}{c}3.5 \\
(0.0-7.5)\end{array}$ & 0.0262 \\
\hline 17.3 & $\begin{array}{c}16.3 \\
(13.9-18.7)\end{array}$ & $\begin{array}{c}18.1 \\
(15.4-20.7)\end{array}$ & $\begin{array}{c}5.1^{* \ddagger} \\
(1.0-9.1)\end{array}$ & $\begin{array}{c}16.5 \\
(14.2-18.8)\end{array}$ & $\begin{array}{c}18.5 \\
(15.9-21.1)\end{array}$ & $\begin{array}{c}3.3^{\star \ddagger} \\
(0.1-6.6)\end{array}$ & $\begin{array}{c}18.2 \\
(15.5-20.9)\end{array}$ & $\begin{array}{c}20.2 \\
(17.3-23.2)\end{array}$ & $\begin{array}{c}2.4^{\star \ddagger} \\
(0.0-5.6)\end{array}$ & $<0.0001$ \\
\hline
\end{tabular}

*significantly less than corresponding value from same survey, $\mathrm{p}<0.05$

‡significantly different from AAD membership data

$95 \% \mathrm{Cl}-95 \%$ Confidence Interval 


\section{SKIN}

Table 2. Practice Demographics Comparing Urban and Rural Respondents to National Data. Across Surveys, similar practice type and levels of experience. Increased urban respondents reporting cosmetic practice compared to rural likely reflecting increased demand as well as natural increase in cosmetic practice since 2014 .

\begin{tabular}{|c|c|c|c|c|c|c|c|}
\hline \multirow[t]{2}{*}{ Demographics } & \multicolumn{2}{|c|}{ Survey 1} & \multicolumn{2}{|c|}{ Survey 2} & \multicolumn{2}{|c|}{ Survey 3} & \multirow{2}{*}{$\begin{array}{c}\text { AAD } \\
\text { Membership }\end{array}$} \\
\hline & Urban & Rural & Urban & Rural & Urban & Rural & \\
\hline \multicolumn{8}{|l|}{$\begin{array}{l}\text { Practice Type } \\
\%(95 \% \mathrm{Cl})\end{array}$} \\
\hline Private & $\begin{array}{c}88.5 \\
(86.5-90.7)\end{array}$ & $\begin{array}{c}92.4 \\
(87.5-97.3)\end{array}$ & $\begin{array}{c}89.1 \\
(87.0-91.2)\end{array}$ & $\begin{array}{c}93.3 \\
(88.8-97.9)\end{array}$ & $\begin{array}{c}89.7 \\
(87.5-92.0)\end{array}$ & $\begin{array}{c}90.6 \\
(84.2-96.9)\end{array}$ & - \\
\hline $\begin{array}{c}\text { University/Academic/ } \\
\text { Government }\end{array}$ & $\begin{array}{c}11.5 \\
(9.3-13.6)\end{array}$ & $\begin{array}{c}7.6 \\
(2.7-12.5)\end{array}$ & $\begin{array}{c}10.9 \\
(8.8-13.0)\end{array}$ & $\begin{array}{c}6.7 \\
(2.1-11.2)\end{array}$ & $\begin{array}{c}10.3 \\
(8.0-12.5)\end{array}$ & $\begin{array}{c}9.4 \\
(3.1-15.7)\end{array}$ & - \\
\hline \multicolumn{8}{|l|}{$\begin{array}{l}\text { Years of Experience } \\
\%(95 \% \mathrm{Cl})\end{array}$} \\
\hline $1-10$ & $\begin{array}{c}21.6 \\
(18.8-24.4)\end{array}$ & $\begin{array}{c}20.3 \\
(12.9-27.7)\end{array}$ & $\begin{array}{c}18.5 \\
(15.9-21.1)\end{array}$ & $\begin{array}{c}21.7 \\
(14.1-29.2)\end{array}$ & $\begin{array}{c}15.0 \\
(12.4-17.6)\end{array}$ & $\begin{array}{c}24.7 \\
(15.3-34.1)\end{array}$ & $27.0 \%$ \\
\hline $11-20$ & $\begin{array}{c}26.0 \\
(23.0-29.0)\end{array}$ & $\begin{array}{c}32.2 \\
(23.6-40.8)\end{array}$ & $\begin{array}{c}25.1 \\
(22.2-28.1)\end{array}$ & $\begin{array}{c}30.8 \\
(22.3-39.3)\end{array}$ & $\begin{array}{c}22.3 \\
(19.2-25.3)\end{array}$ & $\begin{array}{c}25.8 \\
(16.4-35.4)\end{array}$ & $27.5 \%$ \\
\hline $21-30$ & $\begin{array}{c}26.8 \\
(23.8-29.8)\end{array}$ & $\begin{array}{c}23.7 \\
(15.9-31.6)\end{array}$ & $\begin{array}{c}29.8 \\
(26.7-32.9)\end{array}$ & $\begin{array}{c}25.8 \\
(17.8-33.8)\end{array}$ & $\begin{array}{c}30.1 \\
(26.7-33.5)\end{array}$ & $\begin{array}{c}27.1 \\
(17.4-36.7)\end{array}$ & $21.8 \%$ \\
\hline$>30$ & $\begin{array}{c}25.6 \\
(22.6-28.6)\end{array}$ & $\begin{array}{c}23.7 \\
(15.9-31.6)\end{array}$ & $\begin{array}{c}26.5 \\
(23.5-29.5)\end{array}$ & $\begin{array}{c}21.7 \\
(14.1-29.2)\end{array}$ & $\begin{array}{c}32.7 \\
(29.2-36.1)\end{array}$ & $\begin{array}{c}22.4 \\
(13.3-31.4)\end{array}$ & $23.7 \%$ \\
\hline $\begin{array}{l}\text { Practice Mix } \\
\%(95 \% \mathrm{Cl})\end{array}$ & & & & & & & $\begin{array}{l}\text { AAD Practice } \\
\text { Profile } 2017\end{array}$ \\
\hline Medical & $\begin{array}{c}62.8 \\
(61.2-64.4)\end{array}$ & $\begin{array}{c}64.5 \\
(60.7-68.3)\end{array}$ & $\begin{array}{c}60.0 \\
(58.3-61.8)\end{array}$ & $\begin{array}{c}62.2 \\
(58.0-66.4)\end{array}$ & $\begin{array}{c}61.2 \\
(59.5-63.0)\end{array}$ & $\begin{array}{c}62.8 \\
(57.4-68.3)\end{array}$ & $63 \%$ \\
\hline Surgical/Oncology & $\begin{array}{c}26.3 \\
(24.7-27.9)\end{array}$ & $\begin{array}{c}30.2 \\
(26.5-34.0)\end{array}$ & $\begin{array}{c}25.6 \\
(23.9-27.4)\end{array}$ & $\begin{array}{c}27.4 \\
(23.2-31.6)\end{array}$ & $\begin{array}{c}22.8 \\
(21.0-24.5)\end{array}$ & $\begin{array}{c}27.9 \\
(22.5-33.4)\end{array}$ & $25 \%$ \\
\hline Cosmetic & $\begin{array}{c}15.5 \\
(13.9-43.3)\end{array}$ & $\begin{array}{c}9.8 \\
(6.0-21.9)\end{array}$ & $\begin{array}{c}12.0 \\
(10.2-32.3)\end{array}$ & $\begin{array}{c}8.0 \\
(3.7-15.5)\end{array}$ & $\begin{array}{c}13.6 \\
(11.8-37.3)\end{array}$ & $\begin{array}{c}7.0 \\
(1.6-10.2)\end{array}$ & $12 \%$ \\
\hline Dermatopathology & $\begin{array}{c}4.9 \\
(3.3-6.5)\end{array}$ & $\begin{array}{c}1.6 \\
(0.0-5.4)\end{array}$ & $\begin{array}{c}2.4 \\
(0.6-4.1) \\
\end{array}$ & $\begin{array}{c}2.4 \\
(0.0-6.7)\end{array}$ & $\begin{array}{c}2.4 \\
(0.6-4.1)\end{array}$ & $\begin{array}{c}2.2 \\
(0.0-7.6)\end{array}$ & - \\
\hline
\end{tabular}

*Source: American Academy of Dermatology. Practices mix/types not available.

**Source: Margosian E. Medical vs. cosmetic dermatology: Who is doing what?. Dermatology World.2019.

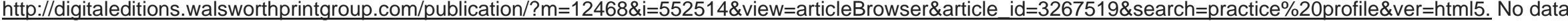
available for dermatopathology

95\% Cl-95\% Confidence Interval 


\section{SKIN}

Table 3. Retrospective (from February to May 2020) and prospective impact of COVID-19 pandemic on dermatologic practices. Urban practices had significantly larger decreases in patient volume and were more likely to be closed during the height of the initial pandemic and saw significantly less patients even as practices began to reopen. Urban practices reporting significantly more sustained use of telemedicine than Rural practice as practices reopened and adapted to new operating procedures.

\begin{tabular}{|c|c|c|c|c|c|c|}
\hline & & $\begin{array}{c}\text { Week of } \\
\text { February 17, } \\
2020\end{array}$ & $\begin{array}{l}\text { Week of } \\
\text { March 16, } \\
2020\end{array}$ & $\begin{array}{c}\text { Week of } \\
\text { April 13, } 2020\end{array}$ & $\begin{array}{c}\text { Week of } \\
\text { May 18, } 2020\end{array}$ & p-value* \\
\hline $\begin{array}{l}\text { How many days } \\
\text { did you practice? } \\
\text { (mean; } 95 \% \mathrm{Cl} \text { ) }\end{array}$ & $\begin{array}{l}\text { Rural } \\
\text { Urban }\end{array}$ & $\begin{array}{c}4.2 \\
(4.0-4.4) \\
4.2 \\
(4.1-4.3)\end{array}$ & $\begin{array}{c}3.4^{\mathrm{A}} \\
(3.0-3.7) \\
3.0^{\mathrm{A}} \\
(2.9-3.2)\end{array}$ & $\begin{array}{c}3.5^{\mathrm{A}} \\
(3.3-3.8) \\
3.5^{\mathrm{A}, \mathrm{B}} \\
(3.4-3.6)\end{array}$ & $\begin{array}{c}3.9 \\
(3.7-4.2) \\
3.6^{\mathrm{A}, \mathrm{B}} \\
(3.5-3.7)\end{array}$ & $<0.0001$ \\
\hline $\begin{array}{l}\text { How many } \\
\text { patients were } \\
\text { seen in your } \\
\text { primary practice } \\
\text { location? } \\
\text { (mean; } 95 \% \mathrm{Cl} \text { ) }\end{array}$ & Urban & $\begin{array}{c}171.3 \\
(139.5-203.1) \\
146.6 \\
(135.9-157.2)\end{array}$ & $\begin{array}{c}85.5^{\mathrm{A}} \\
(68.4-102.5) \\
60.5^{\mathrm{A}} \\
(54.5-66.5)\end{array}$ & $\begin{array}{c}40.0^{\mathrm{A}} \\
(29.4-50.5) \\
26.6^{\mathrm{A}, \mathrm{B}} \\
(23.3-30.0)\end{array}$ & $\begin{array}{c}142.4^{\mathrm{B}, \mathrm{C}} \\
(105.0-179.8) \\
90.9^{\mathrm{A}, \mathrm{B}, \mathrm{C}, \mathrm{D}} \\
(81.6-100.2)\end{array}$ & $<0.0001$ \\
\hline $\begin{array}{l}\text { How many } \\
\text { biopsies did you } \\
\text { perform for } \\
\text { suspicious } \\
\text { pigmented skin } \\
\text { lesions? } \\
\text { (mean; } 95 \% \mathrm{Cl} \text { ) }\end{array}$ & Urban & $\begin{array}{c}20.1 \\
(14.2-25.9)\end{array}$ & $\begin{array}{c}10.5^{\mathrm{A}} \\
(6.4-14.5)\end{array}$ & $\begin{array}{c}2.7^{\mathrm{A}, \mathrm{B}} \\
(2.4-3.1)\end{array}$ & $\begin{array}{c}11.9^{\mathrm{A}} \\
(7.4-16.4)\end{array}$ & $<0.0001$ \\
\hline $\begin{array}{l}\text { Did you } \\
\text { selectively } \\
\text { postpone non- } \\
\text { essential } \\
\text { appointments? } \\
\text { (\%Yes; } 95 \% \mathrm{Cl})\end{array}$ & Urban & $\begin{array}{c}32.3 \\
(22.7-39.7) \\
35.9 \\
(32.1-39.7)\end{array}$ & $\begin{array}{c}68.4^{\mathrm{A}} \\
(57.8-79.1) \\
80.8^{\mathrm{A}} \\
(77.4-84.1)\end{array}$ & $\begin{array}{c}95.8^{\mathrm{A}, \mathrm{B}} \\
(92.2-99.5) \\
100.0^{\mathrm{A}, \mathrm{B}} \\
--\end{array}$ & $\begin{array}{c}68.2^{\mathrm{A}, \mathrm{C}} \\
(58.1-78.3) \\
74.2^{\mathrm{A}, \mathrm{C}} \\
(71.0-77.4)\end{array}$ & $<0.0001$ \\
\hline $\begin{array}{l}\text { How many } \\
\text { biopsies were } \\
\text { postponed? } \\
\text { (mean; } 95 \% \mathrm{Cl} \text { ) }\end{array}$ & $\begin{array}{l}\text { Rural } \\
\text { Urban }\end{array}$ & $\begin{array}{c}3.4 \\
(1.4-5.4) \\
4.0 \\
(3.1-4.9)\end{array}$ & $\begin{array}{c}10.2 \\
(4.9-15.5) \\
10.8^{A} \\
(9.2-12.4)\end{array}$ & $\begin{array}{c}7.5 \\
(4.8-10.2) \\
7.9^{\mathrm{A}, \mathrm{B}} \\
(6.8-9.0)\end{array}$ & $\begin{array}{c}3.8 \\
(1.1-6.6) \\
3.7^{\mathrm{B}, \mathrm{C}} \\
(2.8-4.5)\end{array}$ & $<0.0001$ \\
\hline $\begin{array}{l}\text { Prospective } \\
\text { Estimates }\end{array}$ & & & March 16-20 & April 13-18 & May $18-23$ & p-value ${ }^{* *}$ \\
\hline $\begin{array}{l}\text { If appointments } \\
\text { were postponed } \\
\text { during the week, } \\
\text { when did you } \\
\text { primarily } \\
\text { reschedule them? }\end{array}$ & $\begin{array}{l}\text { Weeks } \\
\text { postponed } \\
\text { (mean; } \\
95 \% \mathrm{Cl}) \\
\% \text { Not } \\
\text { rescheduled } \\
\text { at this time } \\
(\% ; 95 \% \mathrm{Cl})\end{array}$ & $\begin{array}{l}\text { Rural } \\
\text { Urban }\end{array}$ & $\begin{array}{c}5.5 \\
(4.3-6.6) \\
6.5 \\
(6.1-6.8) \\
18.7 \% \\
(0.7 \%-27.7 \%) \\
26.7 \% \\
(23.0 \%-30.5 \%)\end{array}$ & $\begin{array}{c}8.1^{\mathrm{B}} \\
(7.4-8.7) \\
7.5^{\mathrm{B}} \\
(7.2-7.7) \\
19.3 \% \\
(21.1 \%-26.6 \%) \\
19.3 \% \\
(12.1 \%-26.6 \%)\end{array}$ & $\begin{array}{c}4.8^{\mathrm{C}} \\
(3.9-5.8) \\
4.4^{\mathrm{B}, \mathrm{C}} \\
(4.2-4.7) \\
13.1 \% \\
(5.7 \%-20.5 \%) \\
10.7 \% \mathrm{~B}, \mathrm{C} \\
(8.4 \%-12.9 \%)\end{array}$ & $<0.0001$ \\
\hline $\begin{array}{l}\text { If biopsies were } \\
\text { postponed when } \\
\text { did you primarily } \\
\text { reschedule them? }\end{array}$ & $\begin{array}{l}\text { Weeks } \\
\text { postponed } \\
\text { (mean; } \\
95 \% \mathrm{Cl} \text { ) }\end{array}$ & Urban & $\begin{array}{c}7.0 \\
(6.1-7.9) \\
7.2 \\
(6.9-7.5) \\
\end{array}$ & $\begin{array}{c}6.5 \\
(6.0-7.1) \\
6.3^{\mathrm{B}} \\
(6.1-6.6) \\
\end{array}$ & $\begin{array}{c}1.5^{\mathrm{B}, \mathrm{C}} \\
(0.9-2.1) \\
2.0^{\mathrm{B}, \mathrm{C}} \\
(1.8-2.2) \\
\end{array}$ & $<0.0001$ \\
\hline
\end{tabular}

March 2021 Volume 5 Issue 2 


\begin{tabular}{|c|c|c|c|c|c|c|}
\hline & $\begin{array}{l}\% \text { Not } \\
\text { rescheduled } \\
\text { at this time } \\
(\% ; 95 \% \mathrm{Cl})\end{array}$ & $\begin{array}{l}\text { Rural } \\
\text { Urban }\end{array}$ & $\begin{array}{c}65.3 \\
(54.3-76.3) \\
53.8 \\
(49.6-58.1)\end{array}$ & $\begin{array}{c}42.5 \\
(33.5-51.5) \\
36.5^{\mathrm{B}} \\
(33.2-39.7)\end{array}$ & $\begin{array}{c}7.1^{\mathrm{B}, \mathrm{C}} \\
(1.5-12.6) \\
8.8^{\mathrm{B}, \mathrm{C}} \\
\left(6.7^{-10.9)}\right.\end{array}$ & $<0.0001$ \\
\hline \multirow{3}{*}{$\begin{array}{l}\text { Relative to your } \\
\text { practice volume } \\
\text { this week, what } \\
\text { do you } \\
\text { anticipate your } \\
\text { schedule for the } \\
\text { next } 2 \text { weeks will } \\
\text { look like? } \\
\text { (mean } \%: 95 \% \text { Cl) }\end{array}$} & \%Change & $\begin{array}{l}\text { Rural } \\
\text { Urban }\end{array}$ & $\begin{array}{c}-58.3 \\
(-64.9 \text { to }-51.7) \\
-57.3 \\
(-60.1 \text { to }-54.5)\end{array}$ & $\begin{array}{c}-31.4^{\mathrm{B}} \\
(-37.9 \text { to }-24.9) \\
-45.2^{\mathrm{B}, \mathrm{D}} \\
(-47.9 \text { to }-42.4)\end{array}$ & $\begin{array}{c}17.5^{\mathrm{B}, \mathrm{C}} \\
(8.5 \text { to } 26.6) \\
13.0^{\mathrm{B}, \mathrm{C}} \\
\text { (9.6 to } 16.3)\end{array}$ & $<0.0001$ \\
\hline & "Completely & Rural & $\begin{array}{c}7.9 \\
(1.7-14.1)\end{array}$ & $\begin{array}{c}2.5 \\
(0.0-5.4)\end{array}$ & $\begin{array}{c}2.4 \\
(0.0-5.6)\end{array}$ & \\
\hline & $\begin{array}{l}\text { closing } \\
\text { practice" }\end{array}$ & Urban & $\begin{array}{c}20.3 \\
(16.9-23.8)\end{array}$ & $\begin{array}{c}11.9^{\mathrm{B}, \mathrm{D}} \\
(9.7-14.1)\end{array}$ & $\begin{array}{c}3.0^{\mathrm{B}, \mathrm{C}} \\
(1.7-4.2)\end{array}$ & $<0.0001$ \\
\hline $\begin{array}{l}\text { What was your } \\
\text { patient volume } \\
\text { this week } \\
\text { compared to a }\end{array}$ & $\%$ Decrease & $\begin{array}{l}\text { Rural } \\
\text { Urban }\end{array}$ & - & $\begin{array}{c}-68.1 \\
(-72.1 \text { to }-64.0) \\
-71.9 \\
(-73.4 \text { to }-70.4)\end{array}$ & $\begin{array}{c}-35.1^{\mathrm{C}} \\
(-39.6 \text { to }-30.6) \\
-49.4^{\mathrm{C}, \mathrm{D}} \\
(-51.2 \text { to }-47.6)\end{array}$ & $<0.0001$ \\
\hline $\begin{array}{l}\text { typical April } \\
\text { (Survey 2) or May }\end{array}$ & "I was & Rural & - & $\begin{array}{c}5.8 \\
(1.6-10.1)\end{array}$ & $\begin{array}{c}3.5 \\
(0.0-7.5)\end{array}$ & \\
\hline $\begin{array}{l}\text { (Survey 3) week } \\
\text { in your practice? } \\
\text { (mean \%; } 95 \% \mathrm{Cl} \text { ) }\end{array}$ & $\begin{array}{l}\text { closed this } \\
\text { week" }\end{array}$ & Urban & - & $\begin{array}{c}21.4^{\mathrm{D}} \\
(18.6-24.1)\end{array}$ & $\begin{array}{c}8.1^{\mathrm{C}} \\
(6.1-10.1)\end{array}$ & $<0.0001$ \\
\hline $\begin{array}{l}\text { What percentage } \\
\text { of appointments } \\
\text { did you do using } \\
\text { telemedicine (0- } \\
100 \%) ? \\
\text { (Mean \%; } 95 \% \mathrm{Cl})\end{array}$ & & Urban & - & $\begin{array}{c}42.1 \\
(35.5-48.7) \\
\\
49.5 \\
(46.8-52.2)\end{array}$ & $\begin{array}{c}15.1^{\mathrm{C}} \\
(10.3-19.8) \\
\\
27.0^{\mathrm{C}, \mathrm{D}} \\
(24.7-29.3)\end{array}$ & $<0.0001$ \\
\hline $\begin{array}{l}\text { In the next } \\
\text { month, what } \\
\text { percentage of } \\
\text { your patient visits } \\
\text { will be done } \\
\text { using } \\
\text { telemedicine } \\
\text { because of } \\
\text { COVD-19? } \\
\text { (Mean \%; 95\%Cl) }\end{array}$ & & Urban & $\begin{array}{c}38.9 \\
(35.9-41.9)\end{array}$ & $\begin{array}{c}46.5^{\mathrm{B}} \\
(44.2-48.9)\end{array}$ & $\begin{array}{c}21.8^{\mathrm{B}, \mathrm{C}} \\
(19.9-23.7)\end{array}$ & $<0.0001$ \\
\hline 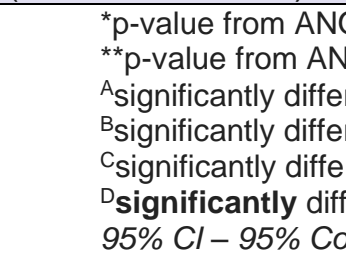 & $\begin{array}{l}\text { from Aprom } 20 \\
\text { nt from value c } \\
\text { lence Interval }\end{array}$ & ne dat & ural/Urban coun & cring on & 05 & \\
\hline
\end{tabular}

noted in urban areas may also reflect increased and continued use of telemedicine, which was nearly nonexistent prior to COVID-19. ${ }^{6}$
Limitations include retrospective estimations and "free-time" bias for Dermatologists with significant decreases in patient volume. However, a consistent and sufficiently large sample size with responses restricted to a 
window of time accounts for these biases. The study period captured a "snapshot" of COVID-19's impact on the US, during which time urban areas had more positive cases and regional COVID-19 impact on recovery rates/clinical practice may parallel regional shifts in COVID-19 caseloads.

Table 4. Urban Versus Rural Predictions for Full ReOpening. Significantly more Rural practices are practicing at full capacity at time of survey (May 2020) compared to Urban practices while a larger proportion of Urban practices predict August or are unsure.

\begin{tabular}{|c|c|c|c|c|}
\hline & & $\begin{array}{c}\text { Urban } \\
\%\end{array}$ & $\begin{array}{c}\text { Rural } \\
\%\end{array}$ & $\begin{array}{c}\text { Mean \% } \\
\text { Difference } \\
\%(95 \% \mathrm{Cl})\end{array}$ \\
\hline \multirow{10}{*}{$\begin{array}{l}\text { When do } \\
\text { you think } \\
\text { your } \\
\text { practice } \\
\text { will } \\
\text { return to } \\
\text { baseline? }\end{array}$} & $\begin{array}{l}\text { Already } \\
\text { back to } \\
\text { baseline }\end{array}$ & 2.0 & 8.2 & $\begin{array}{c}-6.2 \\
(-9.8,-2.7)^{*}\end{array}$ \\
\hline & June & 7.3 & 9.4 & $\begin{array}{c}-2.1 \\
(-5.8,1.5)\end{array}$ \\
\hline & July & 11.5 & 15.3 & $\begin{array}{c}-3.8 \\
(-7.8,0.1)\end{array}$ \\
\hline & August & 13.9 & 8.2 & $\begin{array}{c}5.7 \\
(2.1,9.3)^{*}\end{array}$ \\
\hline & September & 10.5 & 11.8 & $\begin{array}{c}-1.2 \\
(-5.0,2.6)\end{array}$ \\
\hline & October & 6.1 & 5.9 & $\begin{array}{c}0.2 \\
(-3.2,3.5)\end{array}$ \\
\hline & November & 2.4 & 1.2 & $\begin{array}{c}1.3 \\
(-1.1,3.6)\end{array}$ \\
\hline & December & 0.8 & 1.2 & $\begin{array}{c}-0.4 \\
(-2.7,2.0)\end{array}$ \\
\hline & $\begin{array}{c}\text { 1/2021 or } \\
\text { beyond }\end{array}$ & 16.3 & 15.3 & $\begin{array}{c}1.0 \\
(-2.9,5.0)\end{array}$ \\
\hline & Unsure & 29.1 & 23.5 & $\begin{array}{c}5.6 \\
(1.6,9.7)^{*}\end{array}$ \\
\hline
\end{tabular}

\section{CONCLUSION}

These findings demonstrate the significant and differential impact COVID-19 had on urban versus rural dermatology practices. Telemedicine has had a mitigating effect, and may continue to supplement patient volumes as practices recover. However, telemedicine's role and further integration into practice post-pandemic remains uncertain. Further studies may better elucidate the COVID-19's evolving impact on clinical practice in various practice settings. These results provide insight into disparities between practices and can potentially highlight areas for improvement in both practice logistics as well as patient care.

\section{Conflict of Interest Disclosures: None}

Funding: None

\section{Corresponding Author:}

Justin W. Marson, MD

National Society for Cutaneous Medicine

35 E 35th St. \#208

New York NY, 10016

Email: justin.w.marson@gmail.com

\section{References:}

1. Litchman GH, Rigel DS. The immediate impact of COVID-19 on US dermatology practices. J Am Acad Dermatol. 2020;83(2):685-686. doi:10.1016/j.jaad.2020.05.048

2. Litchman GH, Marson JW, Rigel DS. The Continuing Impact of COVID-19 on Dermatology Practice: Office Workflow, Economics and Future Implications. J Am Acad Dermatol. 2020 Sep 30:S0190-9622(20)32658-X. doi:

10.1016/j.jaad.2020.08.131. Epub ahead of print. PMID: 33010326; PMCID: PMC7526524.

3. America is Reopening. But Have We Flattened the Curve? Johns Hopkins University \& Medicine Coronavirus Resource Center. https://coronavirus.jhu.edu/data/new-cases-50states

4. County Rurality Level 2010. US Census Bureau. www2.census.gov/reference/County_Rural_Look up. Accessed 27 October 2020

5. Glazer AM, Rigel DS. Analysis of Trends in Geographic Distribution of US Dermatology Workforce Density. JAMA Dermatol. 2017 May 1;153(5):472-473. doi: 10.1001/jamadermatol.2016.6032. PMID: 28296988; PMCID: PMC5470415.

6. Ehrlich A, Kostecki J, Olkaba H. Trends in dermatology practices and the implications for the workforce. J Am Acad Dermatol. 2017;77(4):746-752. doi:10.1016/j.jaad.2017.06.030. 